\title{
Quantitative classification of WR nuclei of planetary nebulae ${ }^{\star \star \star}$
}

\author{
A. Acker ${ }^{1}$ and C. Neiner ${ }^{1,2}$ \\ 1 Observatoire de Strasbourg, 11 rue de l'Université, 67000 Strasbourg, France \\ 2 RSSD, ESTEC/ESA, Keplerlaan 1, 2201 AZ, Noordwijk ZH, The Netherlands
}

Received 30 October 2002 / Accepted 18 February 2003

\begin{abstract}
We analyse 42 emission-line nuclei of Planetary Nebulae (PNe), in the framework of a large spectrophotometric survey of [WC] nuclei of PNe conducted since 1994, using low/medium resolution spectra obtained at ESO and at OHP. We construct a grid of selected line-intensities (normalized to C IV-5806 $\AA=100$ ) ordered by decreasing ionisation potential going from 871 to $24 \mathrm{eV}$. In this grid, the stars appear to belong clearly to prominent O (hot [WO1-4] types) or C (cooler [WC4-11] types) line-sequences, in agreement with the classification of massive WR stars applied to Central Stars of Planetary Nebulae (CSPNe) by Crowther et al. 1998 (CMB98). We propose 20 selected line ratios and the FWHM of CIV and C III lines as classification diagnostics, which agree well with the 7 line ratios and the $F W H M$ proposed by CMB98. This classification based on ionisation is related to the evolution of the temperature and of the stellar wind, reflecting the mass-loss history. In particular, inside the hot [WO4]-class, we discover four stars showing very broad lines over the whole spectral range. These stars possibly mark the transition from the initial momentum-driven phase to the later energy-driven phase of the CSPNe along their evolution from the post-Asymptotic Giant Branch (post-AGB) phase through [WC] late, [WC4] and [WO]-types. The HR diagram and the diagram linking the terminal velocity and the temperature indicate highly dispersed values of the stellar mass for our sample, around a mean mass higher than for normal CSPNe. The distribution of the 42 stars along the ionisation sequence shows $24 \%$ of [WO1-3], 21\% of [WO4], 17\% of [WC4] hot stars, and 26\% of [WC9-11] cool stars. The [WC5-8] classes remain poorly represented (12\%). This distribution is confirmed on the basis of a large compilation of the 127 known emission-lines CSPNe, which represent about $5 \%$ of the known PNe.
\end{abstract}

Key words. planetary nebulae: general - stars: evolution - stars: AGB and post-AGB - stars: Wolf-Rayet - line: profiles

\section{Introduction}

Among the about 1500 true and probable planetary nebulae listed in Acker et al. (1992 and 1996), a stellar continuum is detectable for about 350 central stars of $\mathrm{PNe}, 30 \%$ of which show carbon (C III-5696 or C IV-5806) emission lines typical for WR stars (the half of them being classified as [WC] see Tylenda et al. 1993; Pena et al. 1998, 2001; Gorny 2001). This means that about $15 \%$ of the observed CSPNe appear as [WC]-stars. However Wolf-Rayet (WR) spectra are easy to detect, even if the stellar continuum is unobservable. In the framework of Parker's recent deep southern $\mathrm{H} \alpha$ survey, about 1000 new PNe were discovered, which are fainter and more extended than in our 1992/96 catalogues, and 11 new [WR] CSPNe were found in a first analysis (see Parker 2002, and Table 4 with 9 of them in this work). In total, the 127 known emission-line

\section{Send offprint requests to: A. Acker,}

e-mail: acker@astro.u-strasbg.fr

* Based on observations obtained at the European Southern Observatory (ESO), La Silla (Chile), and at the Observatoire de HauteProvence (OHP, France).

$\star \star$ Table 4 is only available in electronic form at http://www. edpsciences.org
CSPNe (see Table 4) represent 5\% of the 2500 known PNe. So, we may conclude that 5 to $6 \%$ of the CSPNe show broad emission line-spectra of the WR carbon sequence. Note that the WR stars are relatively more abundant inside the massive stars population where the WR-stage covers about $10^{5}$ years, i.e. 10 or 100 times longer that for the WR-stage of the CSPNe that concerns the predominant low mass stars population.

The majority of the WC CSPNe are distributed among early and late [WC]-subtypes. The cool subclasses of late-[WC] stars seem to closely follow the post-AGB stars, subsequent evolution occuring to hotter, earlier subtypes via the mass-loss process (see e.g. Acker et al. 1996). Only a few stars were found in the [WC5-8] class, a paucity possibly related to a faster evolution through this phase.

In order to make a homogeneous and coherent classification of [WC] CSPNe and contribute to the understanding of the relations between the properties of WR CSPNe along the evolutionary sequence and the emission-lines strengths, we undertake a spectrometric survey of emission-lines CSPNe. In an earlier work, based on the low resolution spectroscopic survey (with a standard 10 min exposure time) performed in 19861991 by Stenholm \& Acker, Tylenda et al. (1993) classified 39 [WC] CSPNe and 38 weak emission-line stars (wels) which 
have the C IV-5806 $\AA$ line systematically weaker and narrower than in the [WC] CSPNe.

Since 1994, we have performed new observations with high signal to noise ratio and better resolution. In a first paper, we analysed 33 stars classified as wels and found that some of them represent an evolutionary link between [WC] stars and PG 1159 stars (see Parthasarathy et al. 1998). The general study we perform on [WR] stars and their PNe is described in Acker \& Durand (2002).

In the present paper we classify 42 [WR] CSPNe into subtypes extending from [WO1] to [WC11]. In Sect. 2, we report on the observations. Section 3 is devoted to the quantitative classification of these stars, followed by a discussion on the link to stellar parameters in Sect. 4. The list of the 127 known emission-lines CSPNe is presented and discussed in Sect. 5.

\section{Observations and line measurements}

The [WC] type CSPNe are selected from the list by Tylenda et al. (1993). We analyse the spectra of 42 CSPNe having the best signal to noise ratio.

\subsection{Telescopes and spectrographs}

Spectra of about 90 CSPNe were obtained in March and July 1994, July 1995, and July 1996, using the Boller and Chivens Spectrograph with a CCD detector on the $1.52 \mathrm{~m}$ telescope at ESO. The spectra cover the wavelength range 370 to $750 \mathrm{~nm}$ with a spectral resolution of 1500 . In order to detect the central star continuum and stellar emission lines, the exposure times were $40 \mathrm{~min}$, leading in most cases to a high signal to noise ratio ( $S / N \geq 30$ for the stellar spectra) (see Partasarathy et al. 1998).

Some additional high-quality spectra were obtained in April 2000, with the EFOSC spectrograph on the $3.6 \mathrm{~m}$ telescope at ESO. With a resolution $R \simeq 800$ and a spectral range from 432 to $600 \mathrm{~nm}$, we adopted exposure times of 15 to $60 \mathrm{~min}$ for the 9 fairly bright CSPNe observed, leading to a value of $S / N \geq 50$.

From 1996 to 2000 we conducted observations of [WC] stars at the "Observatoire de Haute-Provence" (OHP), mainly to study the turbulent outflows from [WC] nuclei (see Grosdidier et al. 2000, 2001, and Acker \& Grosdidier 2002). We used the $1.52 \mathrm{~m}$ telescope equipped with the Aurélie spectrograph with a CCD detector. With a resolution of $R \simeq$ 5000 and a spectral range going from 510 to $600 \mathrm{~nm}$, we adopted exposure times of 15 to $30 \mathrm{~min}$ for the $3 \mathrm{bright}$ CSPNe (PN 64.7+5.0, 120.0+9.8 and 144.5+6.5), leading to a value $S / N \geq 50$. Some low $S / N$ spectra were already obtained in 1993-1994 with the same device for faint CSPNe, using long exposure times ( 2 to 5 hours), and are reported in Acker et al. (1996), and in the thesis of Gorny (1998).

In addition, we used some older observations, in a qualitative way. Photographic plates were taken in 1972 at the $1.52 \mathrm{~m}$ telescope at ESO equipped with the Chilicass spectrograph (see Acker 1976), with good $S / N$ in the blue part of the spectrum showing the O VI doublet for hot stars. Some estimations were also deduced from visual inspection of spectra obtained in $1975 / 76$ on films with the $1.52 \mathrm{~m}$ telescope at OHP equipped with the Pediscou spectrograph, the detector being an image-tube (see Acker 1976). All these qualitative results appear below in Table 4 .

The spectra obtained since 1994 were reduced with the MIDAS software, also applied to the usual instrumental calibration spectra and spectrophotometric standard stars spectra.

Figure 1 (by Dr Sophie Durand for the IAU Symposium 209) presents the spectra of some of the 42 CSPNe selected for this analysis.

\subsection{Measurements}

The measurements of the stellar emission lines in a homogeneous way throughout the whole sample is a complicated task. First of all, the usual diagnostic lines must be measured for all spectra. The C IV-5801/12 $\AA$ doublet is dominant for the hot stars and vanishes progressively. The C III-5696 $\AA$ line is dominant for the coolest CSPNe, whereas C II-4267 $\AA$ and various other $C$ II lines appear. All measurements are complicated by the difficulty of determining the stellar continuum level, in particular in the noisy blue region (due to a poor CCD response). Many lines are very faint and/or blended.

Some lines need special attention:

The OVI-5666 $\AA$ feature is a blend of the OVII-5670 and O VI-5663 lines. The strength of this doublet decreases rapidly.

The O VI-3811/34 A doublet appears noisy and its measurement is uncertain in most cases (see Fig. 1). Therefore, we use this doublet in a qualitative way only, its presence being very important for the classification as a [WO]-type.

The OV-5590 A feature results from blending of several O V lines extended from 5572 to $5608 \AA$ and visible for early types, whereas the weak O III-5592 $\AA$ line appears for late types. Their distinction is difficult on our low resolution spectra. In addition, this feature is severely contaminated by the night sky [O I]-5577 $\AA$ line, whose substraction leads to an amputation of the $\mathrm{O} v$ line in most cases.

The CIV-5801/12 $\AA$ doublet: the intensities of both lines were added even if the doublet is resolved; we are careful concerning the faint C III-5826/36 $⿱$ A doublet growing for late types and blended with the $\mathrm{C}$ IV lines.

The He II-4686 $\AA$ line appears strong on all spectra, but is mixed with the nebular line present for highly excited nebulae. In addition, for early types the line is mixed with the strong C IV-4650 ̊ blend. Reliable measurements are possible only for late-types.

The He II-5412 $\AA$ line, well observed for early types, decreases regularly, but from the [WC4]-type a nearby unidentified line shows increasing values (see Fig. 2). The C IV-5470 line shows similar variations than the He II-5412 line. Probably for these two lines, stellar lines emitted by low ionised species (O III?, C II?) bring an increased contribution.

The $\mathrm{C}$ II lines appear weak and generally blended (blends at $5640 / 62,7231 / 37, \ldots$..). The C II-6578/82 $\AA$ doublet and the C II-7235 $\AA$ line measurements are not reliable, being blended 


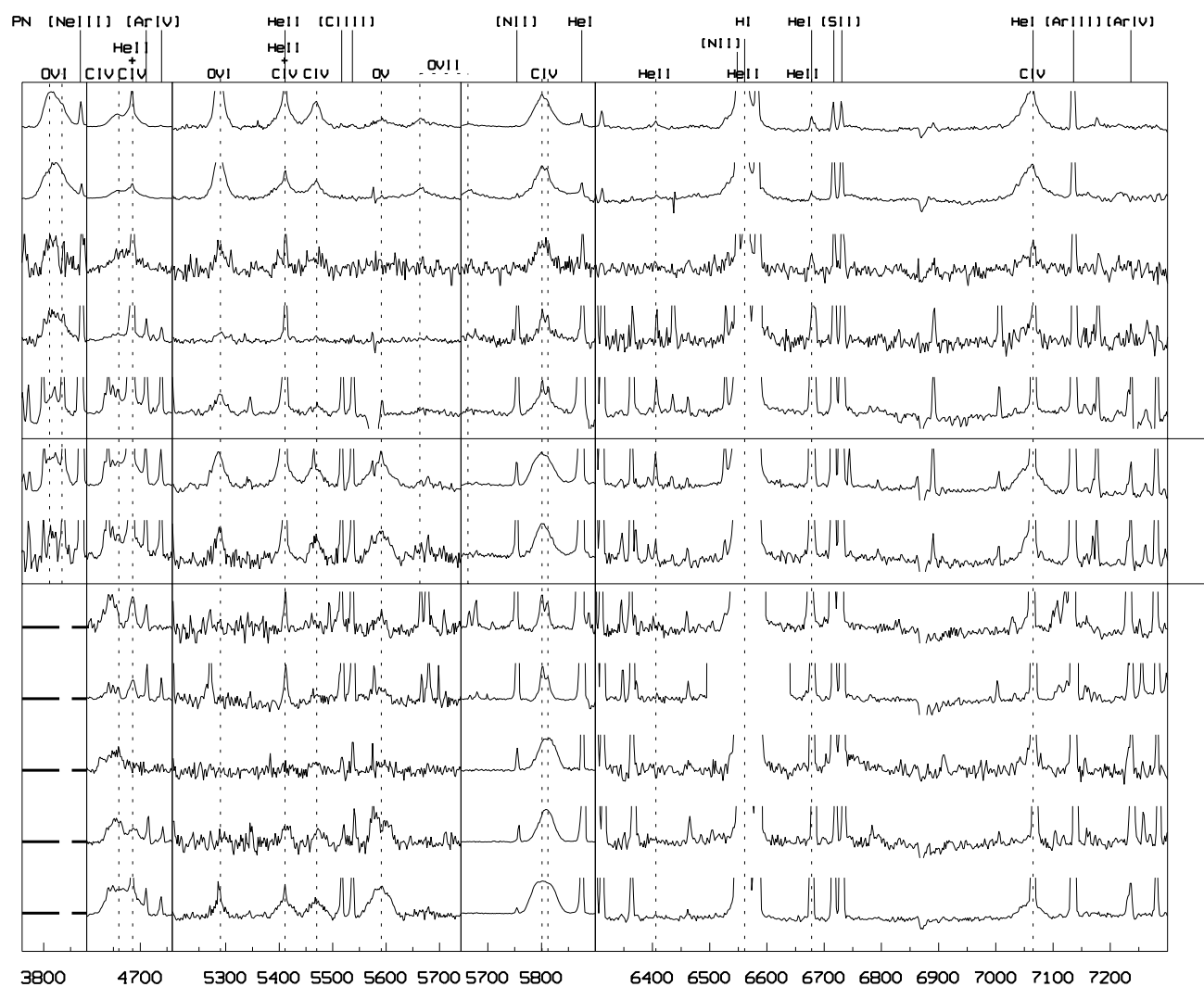

$061.4-09.5$

307. 2-03. 4

$017.9-04.8$

278. $8+04.9$

278. $1-05.9$

358. 3-21. 6

003. $1+02.9$

355. 9-04. 2

019. 4-05. 3

048. $7+01.9$

004. 8-22. 7

336. 2-06. 9

3800

(angstroem)

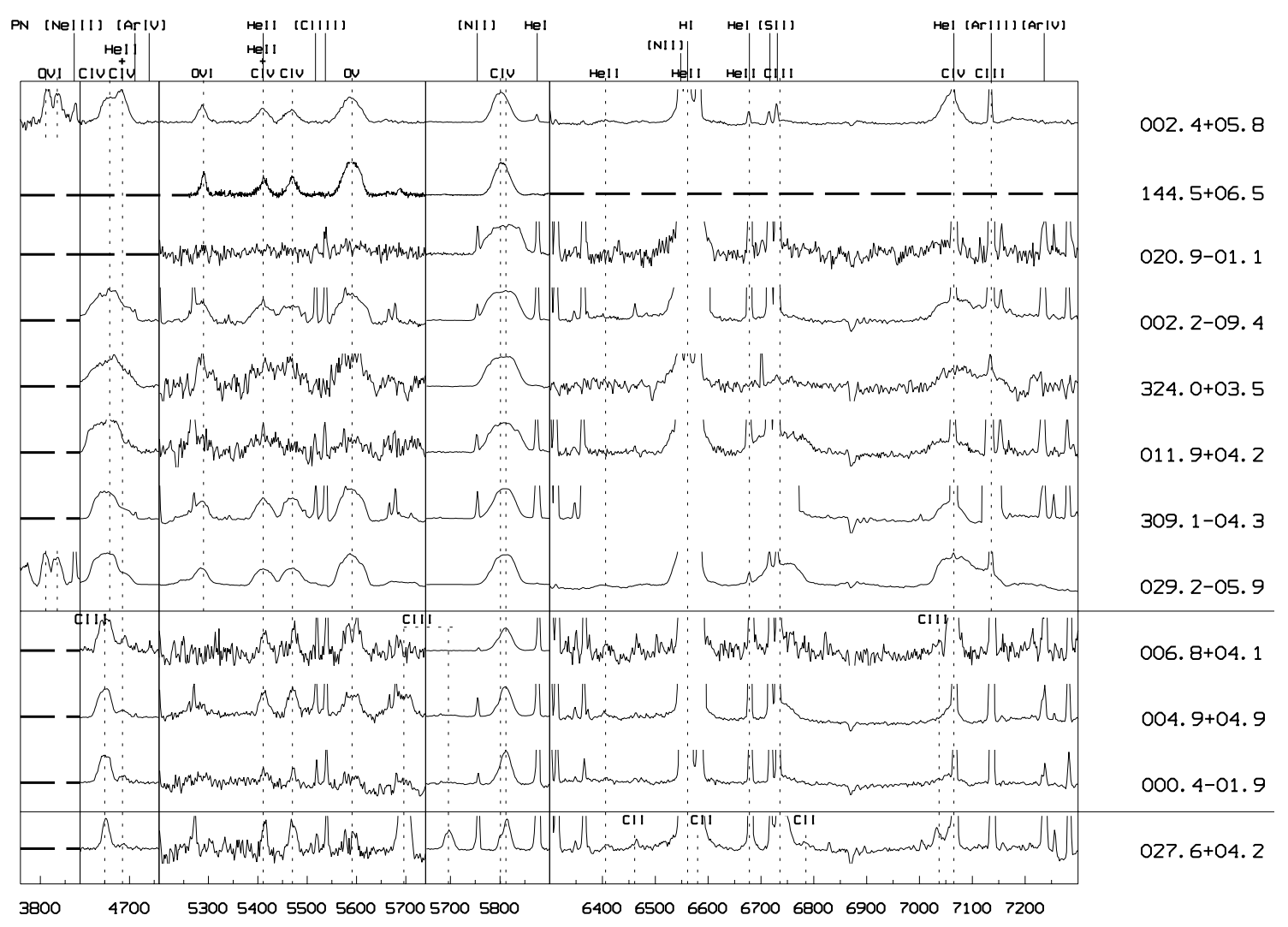

(angstroem)

Fig. 1. Spectra of 36 WR CSPNe stars (see Tables 1a and 3) ordered according to the evolutive sequence. The 5200-5670 $\AA$ range has been zoomed, in order to better distinguish the different emission lines. Nebular lines are shown at the top of the figures. 


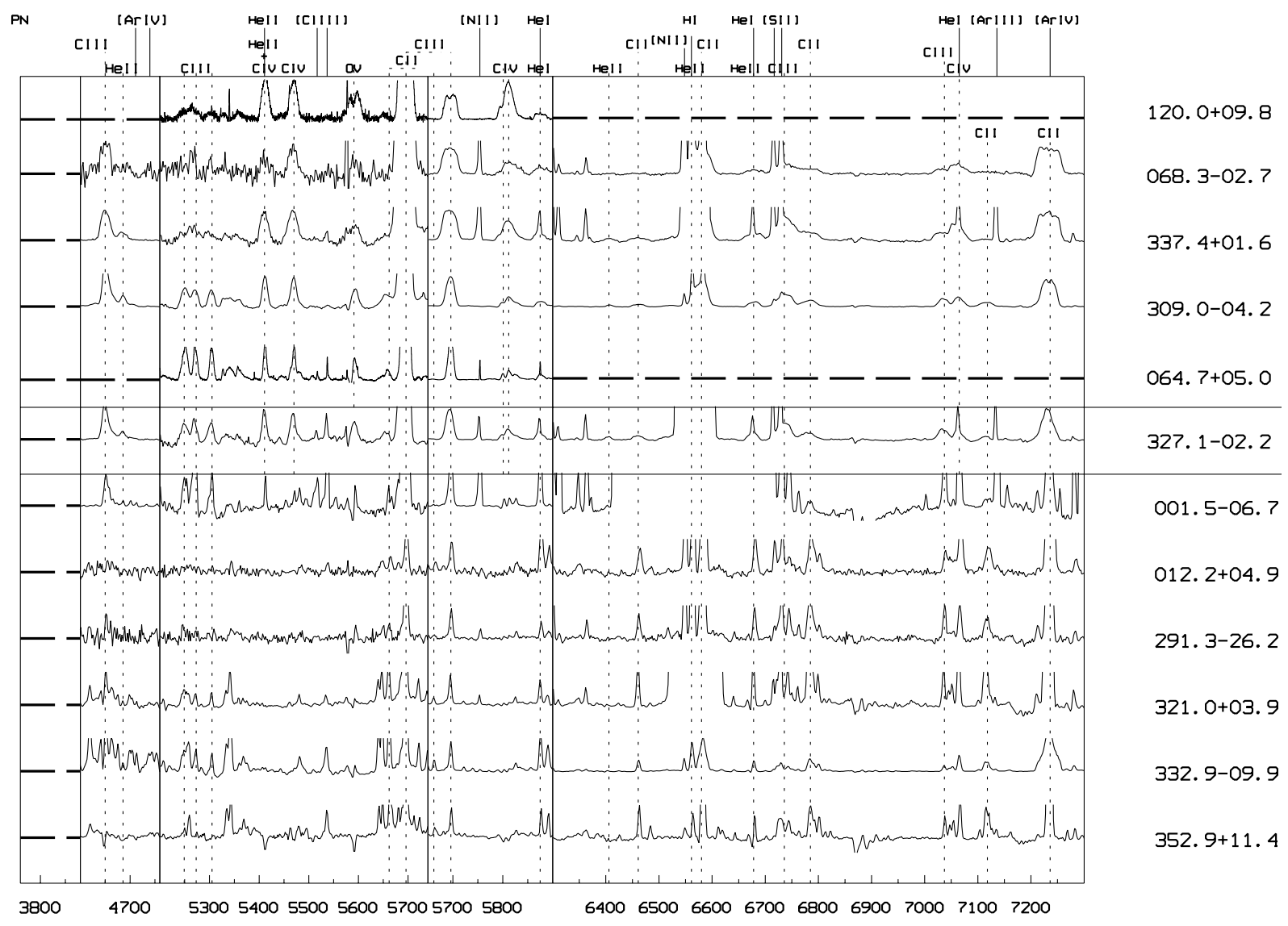

(angstroem)

Fig. 1. continued.

with the nebular lines [N II]-6583 $\AA$ and [Ar IV]-7236 $\AA$ in a different way from one PN to the other.

The line intensities were measured by integration over the line (area in emission over the continuum). In order to perform reliable values, we measured all spectra three times, improving progressively the measurements while discovering the problems listed above. In the case of compact PNe, it is difficult to separate some stellar lines (in particular He II) from nebular ones. The nebular contamination is removed through subtraction of Gaussian fits.

\subsection{Measured and dereddened intensities}

Table 1a presents the measured line intensities, relative to the intensity of the C IV-5801/12 $\AA$ doublet normalized to 100 . We classify the whole set of observed emission lines, according to the corresponding ionisation potential, completed, when available, by the excitation potential (given for the lower atomic level). As shown on the top of Table 1, the highest excitations correspond to oxygen ions ( $\mathrm{O}$ VIII to $\mathrm{O} v$ ), followed by the helium ion (He II), the carbon ions ( $\mathrm{C}$ IV to $\mathrm{C}$ II) corresponding to lower excitations.

Table 1a is organized as follows:

Column 1: PN G numbers as referenced in the "Strasbourg ESO catalogue of galactic Planetary Nebulae" (Acker et al. 1992). The usual names of the PNe are given in Table 4.
PN G 004.8-22.7 (labelled "S") belongs to the Sagittarius dwarf galaxy.

Columns 2 to 22: line intensities relative to C IV-5806 $=100$. On the top, the wavelengths are given in $\AA$ with a mark " $B$ " when the feature is blended. The measurements of lines observed with EFOSC are shown in italics. For the lines observed both with the B\&C and EFOSC spectrographs, we present the weighted mean value, according to the quality of the spectra. All line intensities with an uncertainty $\geq 30 \%$ are marked by a double point ":". A mark "vis" means that the line is detected but the quality of the measurement is very low.

Column 23: intensities measured for the C IV doublet, allowing us to estimate the signal/noise ratio for our spectra, depending on the stellar magnitude and extinction constant (see Table 3). Columns $24 \mathcal{E}$ 25: FWHM measured in $\AA$ for the C IV-5806 and the C III-5696 lines. For the C IV doublet, we give homogeneous values corresponding to the blended line.

Column 26: spectral [WO] or [WC] subtype proposed in this work.

We dereddened the line fluxes by using the extinction constants listed in Table 3 . Table $1 \mathrm{~b}$ presents the dereddened line intensities, relative to the dereddened intensity of the C IV-5806/12 $\AA$ doublet normalized to 100 . Table $1 \mathrm{~b}$ is organized like Table 1a from Cols. 1 to 22 . 
Table 1. a) Measured line intensities, relative to $I(\mathrm{C}$ IV-5806) = 100, and FWHM (C IV-5806 and C III-5696) obtained for 42 WR CSPNe. The symbol "B" indicates that the line results from a blend. The measured values for C IV-5806 are given in Col. 23 (units $10^{-15} \mathrm{ergs} / \mathrm{cm}^{2} / \mathrm{s}$ ). All measurements are based on observations made at the $1.52 \mathrm{~m}$ telescope at ESO $(\lambda \lambda 370-750 \mathrm{~nm})$. Objects marked ${ }^{\circ}$ were also observed at the $3.6 \mathrm{~m}$ telescope at ESO $(\lambda \lambda 432-600 \mathrm{~nm})$, the corresponding measurements are shown in italics. Objects marked * were observed at OHP $(\lambda \lambda 510-600 \mathrm{~nm})$. The symbol "(c)" means that the line was detected by a visual inspection of plates obtained at the $1.52 \mathrm{~m}$ telescope + Chilicass at ESO, in Aug. 1972 (exposure time: 4h). The symbols ":" and "vis" mean respectively that the measurements are of poor quality, and that the line is visible but not measurable. The symbol "A" refers to absorption features.

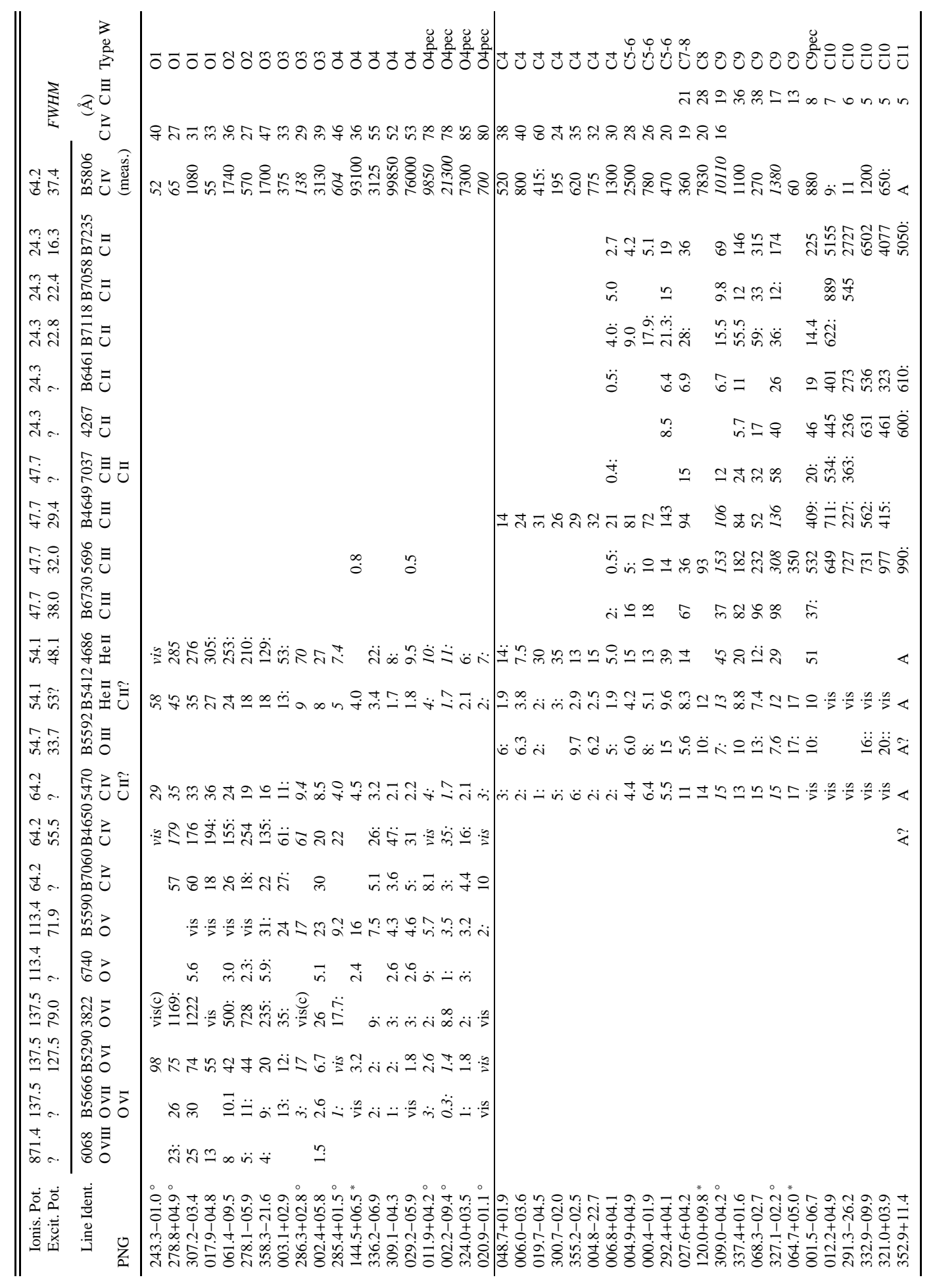


Table 1. b) Dereddened line intensities relative to $I($ C IV-5806 $)=100$, obtained for 42 WR CSPNe.

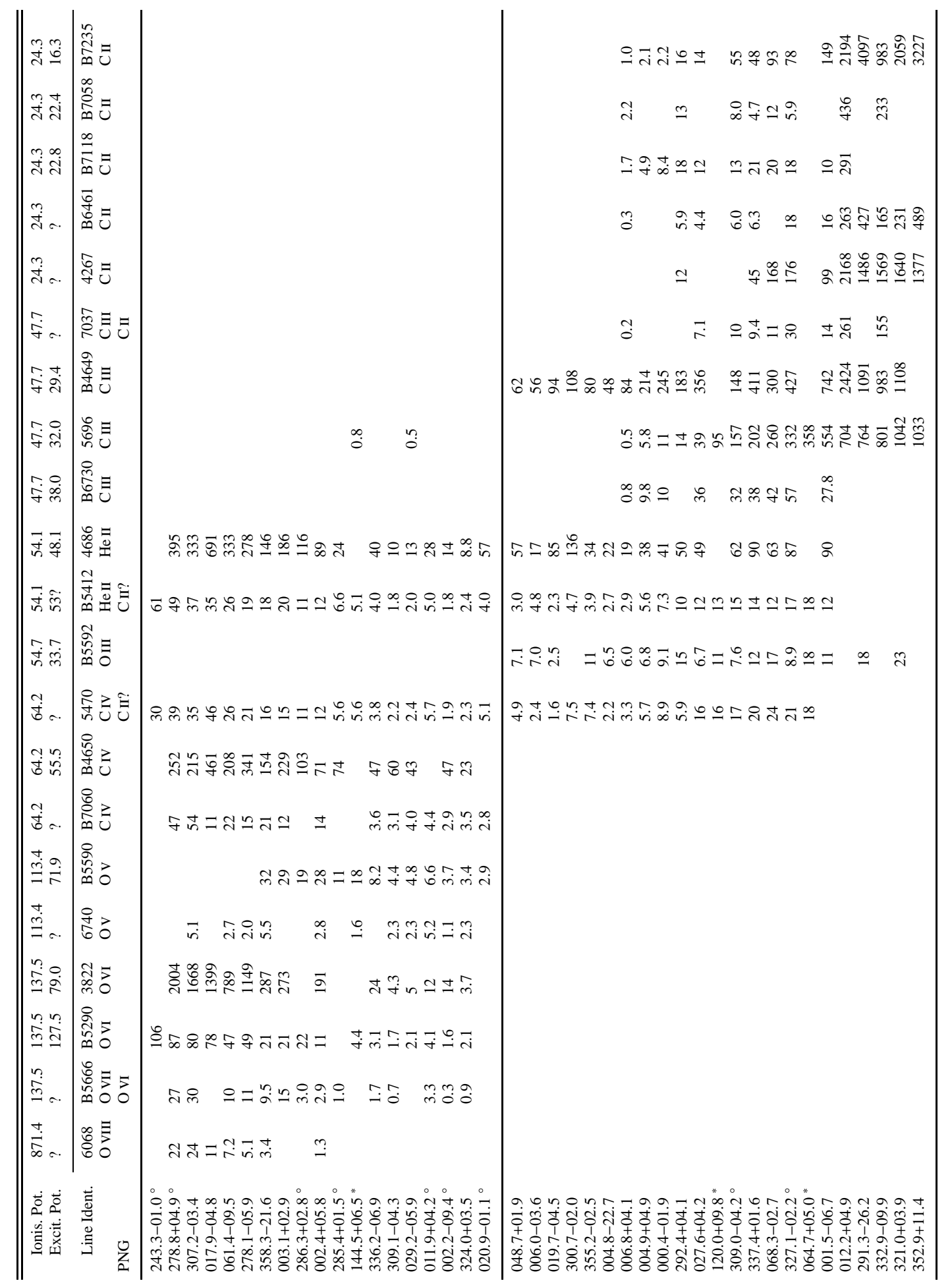

\subsection{Comparison with Crowther et al. (1998)}

We check the reliability of our measurements by comparison with the values presented by Crowther et al. (1998) (CMB98) for $15 \mathrm{CSPNe}, 10$ of which are included in our sample (2 [WO] and 8 [WC8-10], K2-16 being excluded because its C IV-5806 line appears in absorption). Only seven lines were compared, because in the CSPNe sample the HeI-5876 line appears essentially as nebular. The deep spectra by CMB98 $(R=2000$ or 4000 (3 or $1.5 \AA$ ) and $30000(0.2 \AA)$ for some spectra) are comparable to our observations, but our spectrum of He 2-459 (PNG 68.3-2.7) is of low quality and the O VI-3822 and He II-4686 lines are of poor quality in our other spectra. For the two hot [WO] stars, we attribute the 5590 line to $\mathrm{OV}$ and not to $\mathrm{O}$ III, in agreement with the global ionisation level of these stars (see Table 1). 


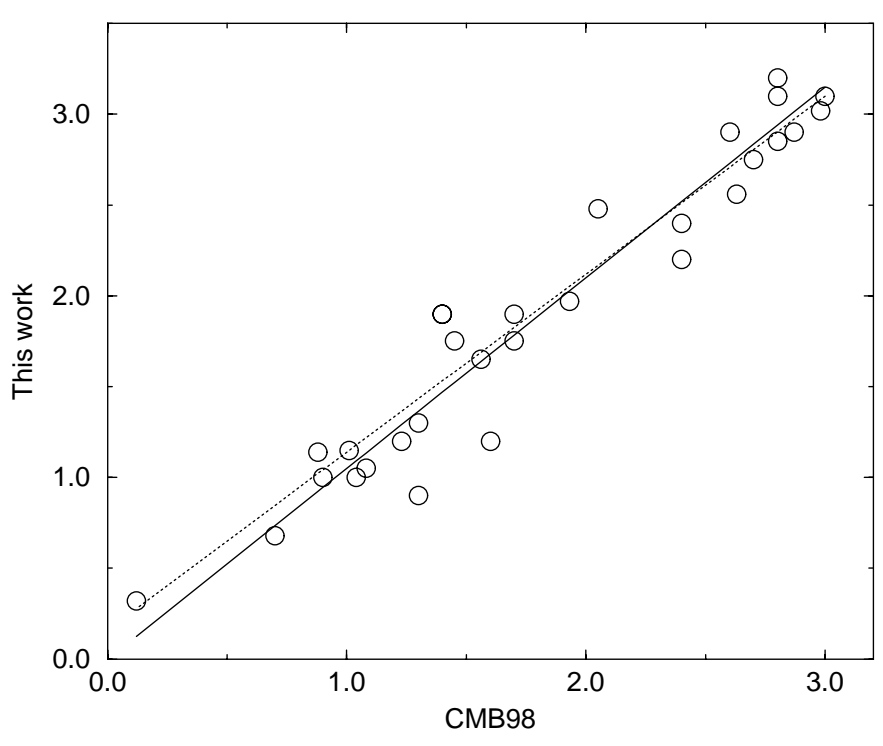

Fig. 2. Comparison of the dereddened line strengths relative to C IV$5806=100$ given in this work and in CMB98. The resulting correlation seems to be linear: this work $=1.05 \times \mathrm{CMB} 98$ with $R^{2}=0.91$ (solid line). An alternative fit gives: this work $=0.98 \times \mathrm{CMB} 98+0.16$ (dotted line).

In order to compare the equivalent widths done by CMB98 to our line intensities (dereddened by using the same extinction constants), we calculate these values relative to (C IV-5806) $=$ 100. Figure 2 shows a good agreement between the two samples, well correlated by a linear relation. The highest discrepancy is noted for the blue O VI-3822 (29.2-5.9) and C II-4267 (68.3-2.7 and 1.5-6.7) lines, and the blended O III/V-5590 line (309.0-4.2 and 332.9-9.9).

\section{Quantitative classification of the observed CSPNe}

\subsection{The classification systems}

After the pioneering work of Smith \& Aller (1969), in which an O VI-sequence with extremely high excitation was introduced, and which lead to the exhaustive catalog by van der Hucht et al. (1981), a systematic classification of WR CSPNe was done by Heap (1982) and Mendez \& Niemela (1982) who defined and characterized the [WC2] to [W11] subtypes. Mendez et al. (1988) and Mendez (1991) found that most of the [WC] CSPNe are helium-rich and hydrogen-deficient. The [WC10-12] cooler extension of the carbon sequence was discussed by Webster \& Glass (1974) and $\mathrm{Hu} \&$ Bibo (1990). CMB98 refined the WC and the WO schemes used for massive WR stars (Torres \& Conti 1986; Smith et al. 1990; Kingsburgh et al. 1995) and defined a unified classification for population I and II WR stars. Recall that massive population I and II WR stars are virtually indistinguishable on the basis of their spectra alone (e.g. CMB98). However, there are two huge differences: (1) the WN types have no certified counterpart among the low mass stars; (2) the WC5-8 range is highly occupied by massive stars and appears as a gap for CSPNe. Therefore good classification criteria allow one to distinguish each subtype in this range for massive stars, but cannot be easily applied to CSPNe.

All these classification systems concerning massive and low mass WR stars are based on the relative strength of the carbon (C II, C III and C IV) and oxygen (O III, O V, O VI, O VII) lines essentially in the 520-590 nm range, used as a diagnostic of the ionisation structure of the stellar atmosphere. As these lines are produced for both types of stars in a region dominated by a fast stellar wind, their intensity may reflect the wind velocity, density and abundance, and the wind temperature goes from about $20000 \mathrm{~K}$ for the [WC11] stars to $100000 \mathrm{~K}$ or even higher for [WC1-2] or [WO1-2] stars.

\subsection{A new classification scheme}

As the ionisation/excitation potentials of the different elements are temperature-dependant, we construct a grid of selected line-intensities ordered by decreasing ionisation potential going from 871 to $24 \mathrm{eV}$. Table 1 is organized following this grid.

We ordered the spectra following decreasing intensities (relative to C IV-5806 = 100) of the most reliable and best represented lines, especially O VI-5290 $\AA$ for the early-types and C III-5696 $\AA$ for the late-types. Following the classification of massive WR stars (Torres \& Conti 1986; Smith et al. 1990; Kingsburgh et al. 1995, CMB98), we attribute the [WO] class to the first sequence, and the [WC] class to the lower part of the diagram.

Table 1 is like a diagram in which two well identified sequences appear: a series of spectra dominated by the highly ionised oxygen lines (hot [WO1-4]-types), and a series dominated by carbon lines (cooler [WC4-11] types).

Our classification scheme is coherent with the CMB98 scheme, but it is (i) based on the global ionisation levels enlarged to the whole (optical) spectrum, and (ii) adapted to the CSPNe specificity even if the stars are faint and the spectra obtained with low resolution. This leads to some differences with the CMB98 scheme:

- Blended 5590 (O III-V) or 4650 (C III-IV) lines can be attributed to a specific ionisation level inside our whole grid.

- The very important O VI-3822 line is strongly affected by interstellar extinction and is very weak or absent for faint or distant CSPNe. We then need to use an alternative criterion: all lines with a comparable ionisation level should be present with specific values for the strongest ones (see Tables 1 and 2). Thus all [WO] subtypes can be separated.

- It is very difficult to separate the subtypes in the range [WC57] by using lines well observed for massive WC stars but which appear very faint for most CSPNe. Note that CMB98 attributed the WC5,6,7 classes only to massive stars.

Table 1 shows the progression of all lines along the WO and WC sequences. Only one object (K2-16) showing many absorption-lines is classified as [WC11]. We remove the [WC12] class, as proposed earlier (Acker et al. 1996 and CMB98).

Table 2 proposes 20 selected line ratios and the $F W H M$ of C IV and C III lines (taken from Table 1b) as classification diagnostics. Our criteria agree well with those given by CMB98, 
Table 2. Classification criteria based on dereddened line intensities relative to C IV-5806 $=100$ for 42 CSPNe. The ratio also studied by CMB98 are shown in italics.

\begin{tabular}{|c|c|c|c|c|c|c|c|c|c|c|c|c|}
\hline WO & $\begin{array}{l}F W H M \\
\text { C IV }\end{array}$ & $\begin{array}{l}\text { O VIII } \\
6068\end{array}$ & $\begin{array}{l}\text { O VII } \\
5666\end{array}$ & $\begin{array}{l}O_{V I} \\
5290\end{array}$ & $\begin{array}{l}O_{V I} \\
3822\end{array}$ & $\begin{array}{l}O V \\
5590\end{array}$ & $\begin{array}{l}\text { C IV } \\
7060\end{array}$ & $\begin{array}{l}\text { C IV } \\
4650\end{array}$ & $\begin{array}{l}\text { C IV } \\
5470\end{array}$ & $\begin{array}{l}\text { He II } \\
5412\end{array}$ & $\begin{array}{l}\text { He II } \\
4686\end{array}$ & $\begin{array}{l}\text { Other } \\
\text { lines }\end{array}$ \\
\hline WO1 & $33 \pm 5$ & $20 \pm 8$ & $>25$ & $>80$ & $>1400$ & - & $35 \pm 20$ & $300 \pm 100$ & $35 \pm 5$ & $45 \pm 15$ & $500 \pm 200$ & O v-6740 present \\
\hline WO2 & $32 \pm 3$ & $6 \pm 1$ & 10: & $48 \pm 2$ & $1000 \pm 200$ & - & $18 \pm 4$ & $270 \pm 60$ & $23 \pm 2$ & $20 \pm 4$ & $300 \pm 30$ & for all [WO] types \\
\hline WO3 & $37 \pm 6$ & $2 \pm 1$ & $8 \pm 6$ & $20 \pm 5$ & $250 \pm 40$ & $27 \pm 5$ & $15 \pm 4$ & $140 \pm 60$ & $14 \pm 2$ & $15 \pm 4$ & $130 \pm 30$ & (rel.int. 2 to 5); \\
\hline WO4 & $52 \pm 6$ & - & 1 & $3 \pm 1$ & $10 \pm 6$ & $9 \pm 5$ & $3.5 \pm .5$ & $55 \pm 10$ & $4 \pm 2$ & $4 \pm 2$ & $25 \pm 15$ & $5696(<1)$ for $[\mathrm{WO} 4]$ \\
\hline WO4pec & $80 \pm 2$ & - & $1.5 \pm 1$ & $3 \pm 1$ & $10 \pm 6$ & $4 \pm 1$ & $3 \pm 1$ & $35 \pm 10$ & $4 \pm 2$ & $3 \pm 2$ & $30 \pm 20$ & and [WO4pec] \\
\hline WC & $\begin{array}{l}F W H M \\
\mathrm{C} \text { IV/III }\end{array}$ & $\begin{array}{l}\text { C III } \\
6730\end{array}$ & $\begin{array}{l}\text { C III } \\
5696\end{array}$ & $\begin{array}{l}\text { C III } \\
4649\end{array}$ & $\begin{array}{l}\text { C III } \\
7037\end{array}$ & $\begin{array}{l}\text { CII } \\
4267\end{array}$ & $\begin{array}{l}\text { C II } \\
6461\end{array}$ & $\begin{array}{l}\text { C II } \\
7118\end{array}$ & $\begin{array}{l}\text { C II } \\
7058\end{array}$ & $\begin{array}{l}\text { C II } \\
7235\end{array}$ & $\begin{array}{l}\text { C III-5696/ } \\
\text { O III-5592 }\end{array}$ & $\begin{array}{l}\text { Other } \\
\text { lines }\end{array}$ \\
\hline WC4 & $37 \pm 8$ & 1: & $<1$ & $70 \pm 20$ & $<1$ & - & $<1$ & 2: & 2: & 1: & $<0.5$ & [WC4] to [WC9]: \\
\hline WC5-6 & $25 \pm 3$ & 10: & $10 \pm 3$ & $210 \pm 30$ & - & 12: & $6:$ & $10 \pm 5$ & 13: & $7 \pm 5$ & $4 \pm 3$ & 5470 (4 to 20$)$ \\
\hline WC7-8 & $22 \pm 3$ & 35: & $60 \pm 30$ & 360: & 7: & - & 4: & 12: & - & 15: & $8 \pm 2$ & 5592 (3 to 20), \\
\hline WC9 & $25 \pm 9$ & $40 \pm 10$ & $260 \pm 100 ?$ & $360 \pm 60$ & $15 \pm 6$ & $130 \pm 50$ & $10 \pm 4$ & $18 \pm 2$ & $8 \pm 4$ & $70 \pm 20$ & $25 \pm 10$ & 5412 (3 to 12 ), \\
\hline WC9pec & 8 & 28 & 550 & 740 & 15 & 100 & 16 & 10 & - & 150 & 110 & 4686 (40 to 90$)$ \\
\hline WC10 & $6 \pm 1$ & - & $850 \pm 150$ & $>1000$ & $200 \pm 60$ & $>1500$ & $270 \pm 80$ & $300:$ & $300 \pm 100$ & $>2000$ & $70 \pm 30$ & [WC11] : abs. lines \\
\hline WC11 & 5 & - & $>1000$ & - & - & $>1400$ & 500: & - & - & $>3000$ & & of He II, O III, C IV \\
\hline
\end{tabular}

who propose only 7 line ratios for the quantification of 12 subclasses. Note that (i) one of their additional criteria is based on the stellar He I-5876 line which is highly dominated by the nebular line and is generally not measurable for CSPNe, and that (ii) the [WC5-8] subclasses cannot be clearly split for CSPNe.

Figure 3 reports the variations for O VI-5290/C IV (earlytypes) and C III-5696/C IV (late-types) dereddened line ratios, relative to $\mathrm{CIV}-5806=100$. The $\mathrm{CIV}-5470 / \mathrm{CIV}$ and He II-5410/C IV line ratios show similar values, with a very good correlation for the [WO1-3] types (see Fig. 4). Note that the He II-5412/C IV-5471 line ratio is related to the $\mathrm{C} / \mathrm{He}$ abundance. The similar aspect of these lines reveals essentially identical $\mathrm{C} / \mathrm{He}$ abundances for our sample of hot [WO] stars. For the [WC4] and later types, the increasing intensity and dispersed values could denote the presence of another low ionised element (C II?, O III?).

For the early WO-types, Fig. 5 (in logarithmic scale) shows that OVI-5290 is increasing with O VII/VI-5666, O VIII-6068, O VI-3822, He II-5412 O V-5590 and C IV-4650 (and CIV-5470, see above). The ratio O VI-3822/He II-5412 and O VI-3822/CIV-5806 selected as classification criteria by CMB98 are well correlated with O VI-3822/O V-5590.

Late-types show the CIII-5696 line intensity increasing with the CIII-4649, C II-4267,-6461,-7235 lines, with He II(C II?)-5410 and C IV(C II?)-5470 lines, but highly dispersed values of O III-5592 are observed (see Fig. 6). A relatively too high value of C III-5696 is shown for SwSt1 for most of these relations. The ratio C III-5696/O III-5592 versus the ratio C III-5696/C IV-5806 (principal classification criterium of CMB98) shows a good correlation along the spectral sequence.

\subsection{Comments on individual CSPNe}

The central star of SwSt1 (1.5-6.7) has been classified as [WC9]pec. The C IV-5806 doublet seems rather peculiar: it is split into three sharp emission components indicating a very low stellar wind compared to that found in normal [WC9] stars. The peculiar properties of this $\mathrm{C}$-rich $\operatorname{star}(\mathrm{C} / \mathrm{O}=4.3$ by mass $)$ and this $\mathrm{O}$-rich nebula ( $\mathrm{C} / \mathrm{O}$ close to solar) could indicate a recent origin for the WC spectrum and a O-rich AGB progenitor, contrary to other [WC9] stars which show a nebular C/O ratio $=7$ indicating C-rich AGB progenitors (see de Marco et al. 2001).

The case of the extensively studied PN NGC4O (120.0+9.9) is rather puzzling: this very low excitation planetary nebula (nebular excitation class 3) is excited by a hot [WC8]-type nucleus with a rather low Zanstra $\mathrm{Tz}(\mathrm{HI})$ temperature $(\simeq 30 \mathrm{kK})$, but much higher values are evaluated, e.g. in the range [70-80] kK (Hamann 1996), and reach $90 \mathrm{kK}$ from IUE spectra analysis (Feibelman 1999).

The [WO1-2] nuclei of NGC 2867 (278.1-05.9) and PB 6 (278.8+04.9) show weak and narrow absorption feature around $5580 \AA$. These stars may be in transition phase towards PG 1159-type stars (see Parthasarathy et al. 1998, for more details).

Peculiar [WO4] stars: four [WO4] stars present very large lines over the whole spectrum, with a mean FWHM for the CIV-5806 $\AA$ line of $80 \AA$ denoting a very high terminal velocity and perhaps a contribution of He II or other elements (Fig. 7). We classify them as [WO4]pec stars. Similar cases have been found earlier. Torres et al. (1986) noted that two WC5-7 (WR125 and WR140) show very broad lines, and propose that they are non-thermal emitters with an opaque stellar wind in the radio range, and bright X-ray emission. 


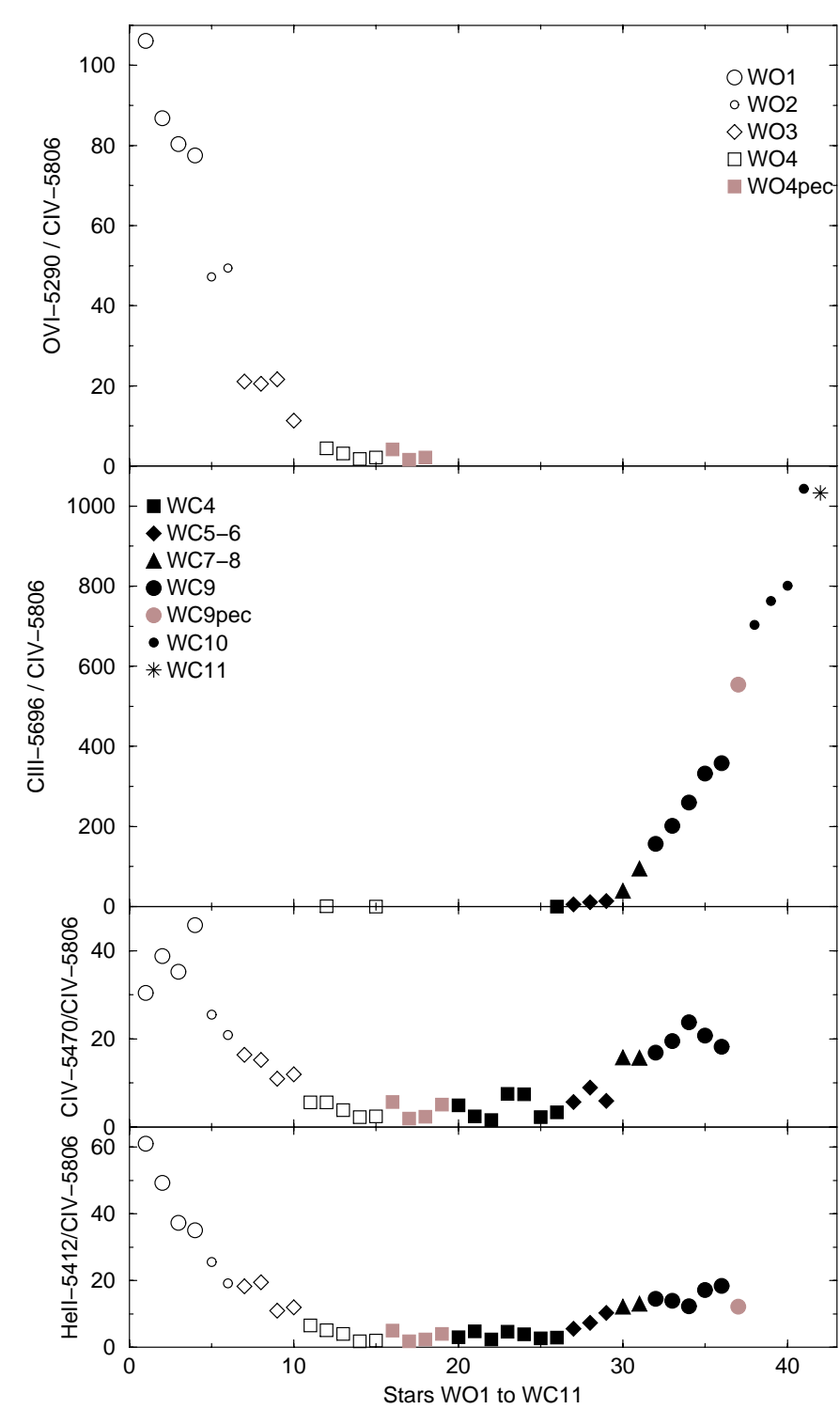

Fig. 3. Upper panels: variation along the $[\mathrm{WO}]$ and $[\mathrm{WC}]$ types of the O VI-5290/C IV-5806 (early-types) and C III-5696/C IV-5806 (latetypes) dereddened line ratios. Lower panels: variation of the CIV5470/C IV-5806 and He II(C II?)-5412/C IV-5806 dereddened line ratios. The numbers 1 to 42 on the abscissa correspond to the observed stars (see Table 3). All line intensities are relative to C IV-5806 $=100$.

Grafener et al. (1998) measured very high mass-loss and terminal velocities $\left(2800 \mathrm{~km} \mathrm{~s}^{-1}\right)$ for three luminous massive WC4 LMC stars (BAT99-11, -52, -61).

\section{The effect of stellar temperature and stellar wind on the [WO]-[WC] classification}

We search for possible correlation between stellar parameters and strenghts of emission-lines used as classification criteria. In particular, we try to understand why the classification of [WC5-8] stars is such a difficult task.

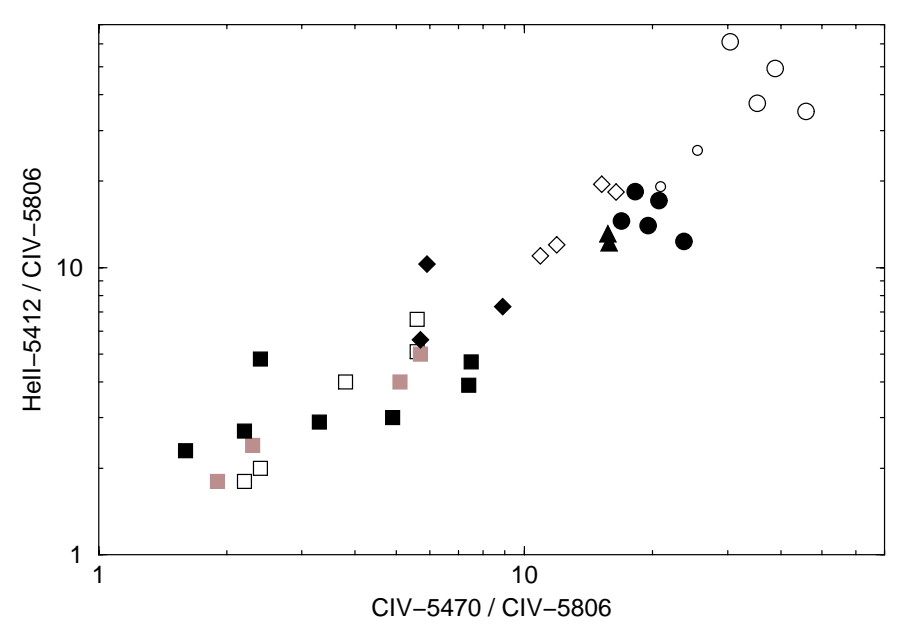

Fig. 4. Correlation between the CIV-5470/C IV-5806 and He II5410/C IV-5806 dereddened line ratios. All line intensities are relative to C IV-5806 = 100. See symbol legend in Fig. 3 .

The main known parameters for the analysed objects are shown in Table 3 as follows:

Column 1: object number from 1 to 42 .

Column 2: PN G number.

Column 3: [WO]-[WC] type from this work.

Column 4: stellar $V$ magnitudes from Acker et al. (1992), CMB98.

Column 5: optical extinction coefficients, taken from CMB98 $(c=1.46 \times 1.21 \times E(b-v))$ and Tylenda et al. (1992).

Columns 6, 7, 8 and 9: respectively, the Zanstra (Gleizes et al. 1989), energy-balance (Preite-Martinez et al. 1989 and 1991) and model atmosphere (Hamann 1996; Koesterke \& Hamman 1997; Leuenhagen \& Hamann 1998; de Marco et al. 2001 and 1998 for objects 37, 40 and 41; Dudziak et al. 2001 for object 25) temperatures, and the adopted value of the temperature $(\log T)$.

Columns 10 and 11: respectively, the terminal velocity obtained by modelling the spectra (Koesterke \& Hamann 1997b; de Marco et al. 2001 and 1997 for objects 37, 40 and 41) and wind velocity deduced from this work: $52 \times 1.2 \times F W H M(\mathrm{C}$ IV $)$ and $52 \times F W H M(\mathrm{C}$ III $)$.

Column 12: distance in kpc from Bensby \& Lundstrom (2001) (BL01), Dudziak et al. (2001) (DPZ01), Van de Steene \& Zijlstra (1994) (vdZ94), Zhang (1995) (Z95), and Acker et al. (1992). For the faint [WC10] objects, the authors propose a very approximate distance in relation to their apparent magnitude.

\subsection{The temperature}

The continuum of a WR star is formed in the wind and depends on the wavelength. Therefore the effective temperature is badly defined. A Zanstra $\mathrm{Tz}(\mathrm{HI})$ temperature is known for 25 of the 42 stars. $\mathrm{Tz}(\mathrm{HI})$ decreases from about 100000 for [WO1-3] stars to 20000 for [WC10] stars. For hot stars, $\mathrm{Tz}(\mathrm{HI})$ is an underestimate of the effective temperature, which is close to the He II Zanstra temperature (see Gleizes et al. 1989). More reliable temperatures could be determined by 
Table 3. Stellar parameters of the 42 analysed CSPNe. A mean value of the stellar temperatures and $V \infty$ is given for each subtype. For SwSt 1 (PN G 1.5-6.7) the mark * refers to the text. Uncertain and very uncertain values are followed by ":" and "::" respectively.

\begin{tabular}{|c|c|c|c|c|c|c|c|c|c|c|c|}
\hline \multirow[b]{2}{*}{$\#$} & \multirow[b]{2}{*}{ PN G } & \multirow[b]{2}{*}{ Type } & \multirow[b]{2}{*}{$v(\mathrm{mag})$} & \multirow[b]{2}{*}{$c(\mathrm{H} \beta)$} & \multicolumn{3}{|c|}{ Stellar temp. (kK) } & \multirow{2}{*}{$\begin{array}{l}\text { adopted } \\
\log T\end{array}$} & \multicolumn{2}{|c|}{$V \infty\left(\mathrm{km} \mathrm{s}^{-1}\right)$} & \multirow[b]{2}{*}{$D(\mathrm{kpc})$} \\
\hline & & & & & $T_{z}(\mathrm{HI})$ & $T_{\mathrm{eb}}$ & $T_{\mathrm{am}}$ & & Model & This work & \\
\hline 1 & $243.3-01.0$ & WO1 & & 0.29 & 81.3 & & 141.0 & & 3000 & 2496 & 3.22 (BL01) \\
\hline 2 & $278.8+04.9$ & WO1 & 17.6 & 0.52 & & 102.7 & 140.0 & 5.01 & 3000 & 1685 & $4.16(\mathrm{vdSZ} 94)$ \\
\hline 3 & $307.2-03.4$ & WO1 & 14.3 & 0.30 & 69.2 & & 135.0 & & 3000 & 1934 & 1.27 (BL01) \\
\hline \multirow[t]{2}{*}{4} & $017.9-04.8$ & WO1 & 16.0 & 1.30 & 34.7: & 49.0: & & 4.69 & & 2059 & $5.62(\mathrm{vdSZ} 94)$ \\
\hline & & & & & 62 & 76 & 139 & 4.85 & 3000 & 2044 & \\
\hline 5 & $061.4-09.5$ & WO2 & 14.6 & 0.45 & 66.1: & 58.9: & 141.0 & 4.77 & 1800 & 2246 & 2.2 vdSZ94) \\
\hline \multirow[t]{2}{*}{6} & $278.1-05.9$ & WO2 & 15.7 & 0.44 & 128.8 & 112.2 & 141.0 & 5.05 & 1800 & 1685 & 1.91 (BL01) \\
\hline & & & & & 97 & 86 & 141 & 4.91 & 1800 & 1966 & \\
\hline 7 & $358.3-21.6$ & WO3 & 16.6 & 0.19 & 91.2 & 91.2 & & 4.96 & & 2933 & 3.76 \\
\hline 8 & $003.1+02.9$ & WO3 & $>17.0$ & 1.99 & 63.1 & 85.1 & & 4.93 & & 2059 & 2.60(vdSZ94) \\
\hline 9 & $286.3+02.8$ & WO3 & 17.4 & 0.80 & & 74.1: & 128.0 & 4.87 & 3000 & 1810 & 2.83 (BL01) \\
\hline \multirow[t]{2}{*}{10} & $002.4+05.8$ & WO3 & 15.9 & 1.93 & 63.1 & 83.2 & 150.0 & 4.92 & 1200 & 2434 & 0.72 (vdSZ94) \\
\hline & & & & & 72 & 83 & 139: & 4.92 & 2100 & 2309 & \\
\hline 11 & $285.4+01.5$ & WO4 & & 1.87 & & 85.1 & & 4.93 & & 2870 & 3.75 (Z95) \\
\hline 12 & $144.5+06.5$ & WO4 & 14.5 & 1.20 & 40.7 & & 134.5 & & 1800 & 2246 & 1.20 \\
\hline 13 & $336.2-06.9$ & WO4 & 16.5 & 0.93 & 52.5 & 72.4 & & 4.86 & & 3432 & $4.76(\mathrm{vdSZ} 94)$ \\
\hline 14 & $309.1-04.3$ & WO4 & 14.4 & 0.36 & 67.6 & 64.6 & & 4.81 & & 3245 & 2.56 (BL01) \\
\hline \multirow[t]{2}{*}{15} & $029.2-05.9$ & WO4 & 14.6 & 0.5 & 50.1 & & 135.0 & & 1600 & 3307 & 1.96 (BL01) \\
\hline & & & & & 53 & 74 & 135 & 4.87 & 1700 & 3020 & \\
\hline 16 & $011.9+04.2$ & WO4pec & 17.0 & 1.59 & & & & & & 4867 & 3.44 (vdSZ94) \\
\hline 17 & $002.2-09.4$ & WO4pec & 16.6 & 0.44 & 69.2 & 109.6 & & 5.04 & & 4867 & 4.4 (vdSZ94) \\
\hline 18 & $324.0+03.5$ & WO4pec & $13 ?$ & 0.57 & & & & & & 5304 & $4(Z 95)$ \\
\hline \multirow[t]{2}{*}{19} & $020.9-01.1$ & WO4pec & $>18$ & 3.30 & & 96.2 & & 4.98 & & 4992 & 1.75 \\
\hline & & & & & 69: & 103 & & 5.01 & & 5008 & \\
\hline 20 & $048.7+01.9$ & WC4 & $>17$ & 2.30 & & & & & & 2371 & 4.37 (Z95) \\
\hline 21 & $006.0-03.6$ & WC4 & & 1.30 & & & & & & 2496 & 4.75 (vdSZ94) \\
\hline 22 & $019.7-04.5$ & WC4 & & 1.65 & & 74.1 & & 4.87 & & 3744 & $5.50(\mathrm{vdSZ} 94)$ \\
\hline 23 & $300.7-02.0$ & WC4 & 18.4 & 2.17 & 63.1 & 61.7 & & 4.79 & & 1498 & 3.53 (vdSZ94) \\
\hline 24 & $355.2-02.5$ & WC4 & & 1.53 & & 77.6 & & 4.89 & & 2184 & \\
\hline 25 & $004.8-22.7$ & WC4 & & 0.60 & 61.0 & 91.2 & 89 & 4.96 & & 1997 & 25(DPZ01) \\
\hline \multirow[t]{2}{*}{26} & $006.8+04.1$ & WC4 & & 2.10 & & 55.0 & & 4.74 & & 1872 & 3.92 (vdSZ94) \\
\hline & & & & & 62 & 72 & 89: & 4.85 & & 2309 & \\
\hline 27 & $004.9+04.9$ & WC5-6 & 17.7 & 1.46 & 61.7 & 56.2 & & 4.75 & & 1747 & 4.81 (vdSZ94) \\
\hline 28 & $000.4-01.9$ & WC5-6 & 16.1 & 1.85 & & 45.7 & & 4.66 & & 1622 & \\
\hline \multirow[t]{2}{*}{29} & $292.4+04.1$ & WC5-6 & 13.8 & 0.38 & 31.6 & 33.1: & & 4.52 & & 1248 & $5.5(\mathrm{vdSZ} 94)$ \\
\hline & & & & & 47 & 45 & & 4.64 & & 1539 & \\
\hline 30 & $027.6+04.2$ & WC7-8 & & 2.00 & & 74.1 & 77.0 & 4.87 & 850 & 1139 & 3.74 (Z95) \\
\hline 31 & $120.0+09.8$ & WC8 & 11.8 & 0.6 & 30.2 & & 78.0 & 4.89 & 1000 & 1352 & $1.12(\mathrm{vdSZ} 94)$ \\
\hline & & & & & $30:$ & $74:$ & 78 & 4.88 & 925 & 1246 & \\
\hline 32 & $309.0-04.2$ & WC9 & 13.3 & 0.52 & 25.7 & 41.5 & 49.0 & 4.53 & 900 & 993 & 4.36(vdSZ94) \\
\hline 33 & $337.4+01.6$ & WC9 & $>17.1$ & 2.40 & 39.8 & & & 4.60 & & 1872 & 2.60 \\
\hline 34 & $068.3-02.7$ & WC9 & 17.3 & 2.65 & & & 77.0 & & 1000 & 1976 & 3.35 \\
\hline 35 & $327.1-02.2$ & WC9 & 15.7 & 1.73 & 43.7 & 25.7 & & 4.54 & & 884 & 3.85 (BL01) \\
\hline 36 & $064.7+05.0$ & WC9 & 10.3 & 0.52 & 35.5 & & 47.0 & 4.55 & 700 & 676 & 1.84 (vdSZ94) \\
\hline & & & & & 36 & 34 & 58 & 4.56 & 867 & 1280 & \\
\hline 37 & $001.5-06.7$ & WC9pec & 11.8 & 0.90 & 35.5 & 26.3 & 40 & 4.49 & $400^{*}$ & 420 & 3.8 (vdSZ94) \\
\hline 38 & $012.2+04.9$ & WC10 & 16.0 & 1.86 & & & 35.0 & & 360 & 364 & $6::$ \\
\hline 39 & $291.3-26.2$ & WC10 & 15.8 & 2.21 & & & 32.0 & & 225 & 260 & $6::$ \\
\hline 40 & $332.9-09.9$ & WC10 & 10.9 & 1.00 & 17.0 & & 21.7 & 4.23 & 225 & 312 & $1.5::$ \\
\hline 41 & $321.0+03.9$ & WC10 & 11.9 & 1.48 & 21.4 & & 16.4 & 4.33 & 160 & 260 & $1.5::$ \\
\hline 42 & $352.9+11.4$ & WC11 & 12.9 & 0.97 & & 19.5 & 30.0 & 4.29 & 300 & 260 & $5::$ \\
\hline & & & & & 19 & 19: & 27 & 4.28 & 226 & 291 & \\
\hline
\end{tabular}



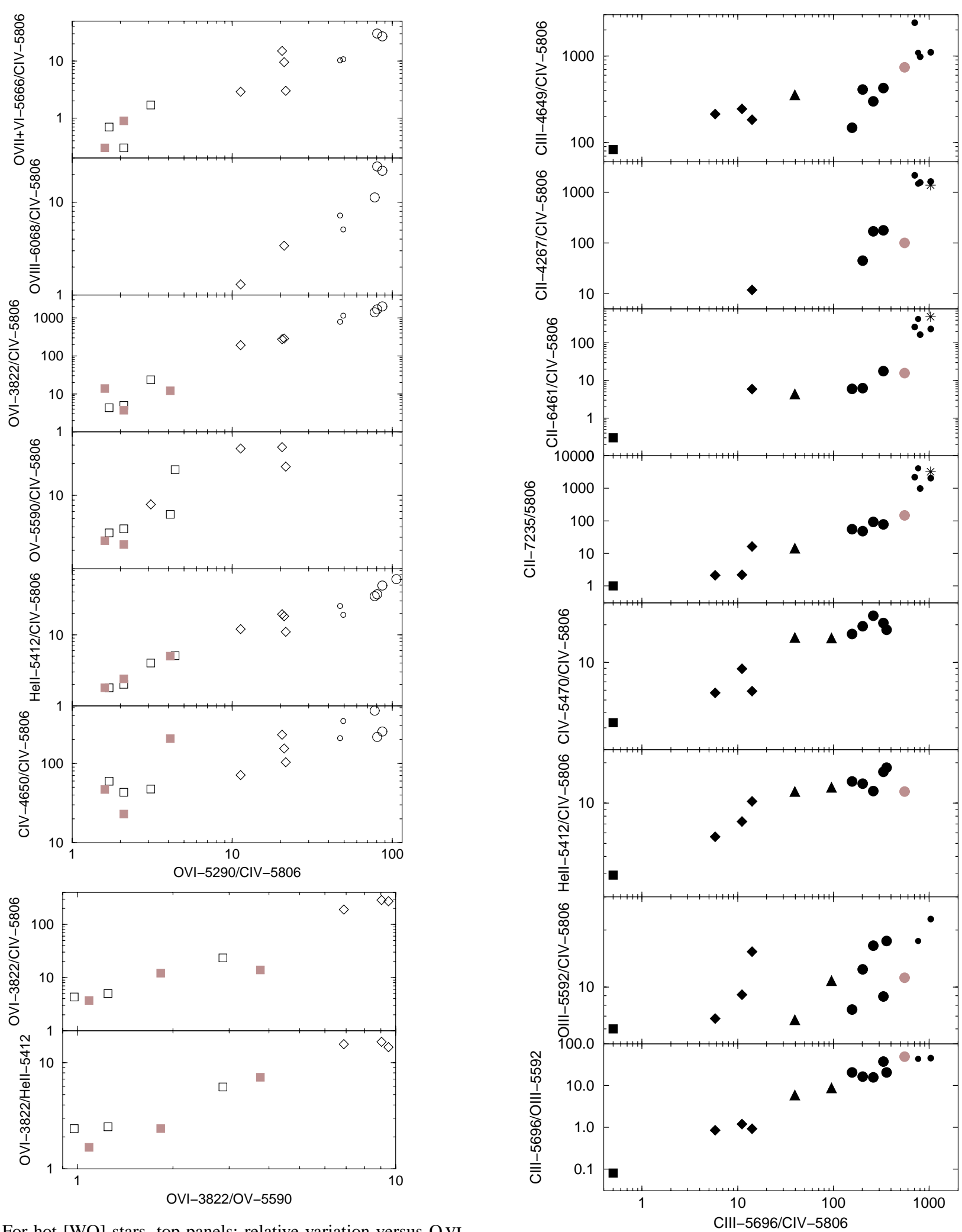

Fig. 5. For hot [WO] stars, top panels: relative variation versus O VI -5290 of the O VII+VI-5666, O VIII-6068, O VI-3822, O V-5590, He II5412 and C IV-4650 dereddened line intensities. Bottom panels: relative variation versus O VI-3822/O V-5590 of the O VI-3822/C IV-5806 and O VI-3822/He II-5412 dereddened line intensities. All line intensities are relative to $\mathrm{C}$ IV-5806 $=100$. See symbol legend in Fig. 3 .

Fig. 6. For [WC] stars, relative variation versus C III-5696 of the C III4649, C II-4267,-6461,-7235, C IV(C II?)-5470, He II(C II?)-5412 and O III-5592 dereddened line intensities, and of the C III-5696/O III-5592 ratio. All line intensities are relative to C IV-5806 $=100$. See symbol legend in Fig. 3. 


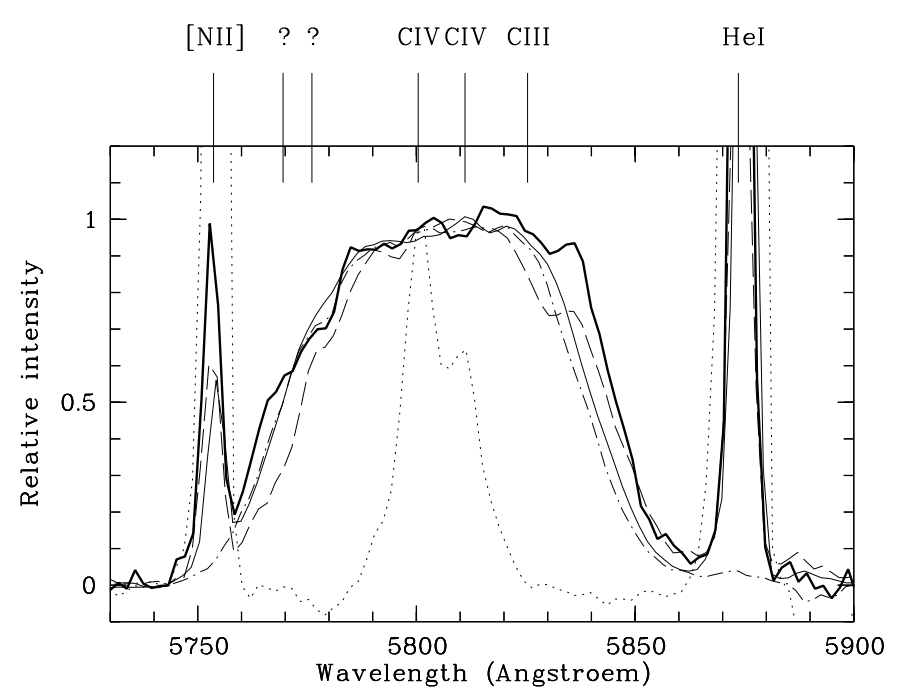

Fig. 7. C IV-5801/12 superimposed line profiles of the four [WO4]pec stars. The thick profile corresponds to M 1-51 (20.9-1.1), and the thin solid line to Cn 1-5 (2.2-9.4). M 1-32 (11.9+4.2) and PM 1-89 $(324.0+3.5)$ are represented with a dashed and dashed-dotted line respectively. For comparison, the C IV line profile of the wels star M 161 (19.4-5.3) has been added (dotted line).

using the "energy-balance method" (done by Preite-Martinez et al. by using ICF) or methods based on stellar models better adapted to CSPNe than with a black body assumption (see ref. in the precedent subsection). We adopt the most reliable value for each object.

We confirm the general trend for the stellar temperature to increase along the [WC]-[WO] evolutionary sequence, without a gap for the [WC5-8] stars (see Fig. 8). But temperature is not an essential criterium to discriminate the [WC5-8] stars. As proposed by Crowther (1999) the main criterium is related to the stellar wind.

\subsection{The stellar wind}

The WC emission lines reflect the parameters of the stellar wind: amount, velocity and clumping. For WR stars, the terminal velocity $V \infty$ could be deduced from the $F W H M$ of the emission lines, as these lines are formed over a very extended region of the high density wind. The C III line is located furthest out and may have velocities closest to the terminal velocity, which is not the case of higher excitation lines. For some [WC7-9] stars the FWHM is measured for the C IV-5808 and the C III5696 lines, by us (Table 1a) and by CMB98, leading to a ratio $\mathrm{C}$ III/C IV $=1.2$. We apply this factor to all $F W H M$ (C IV). Thus the wind velocity $V \infty=62.4 \times F W H M(\mathrm{C}$ IV) for objects 1 to 30 and $52 \times F W H M$ (C III) for objects 31 to 42 (Col. 11 of Table 3). Unfortunately, only the C III and C IV lines have measurable profiles for the whole sample.

Our estimations fit well with the values calculated by model atmospheres for early [WO1-3] and late [WC8-10] CSPNe. However, for [WO4] stars measured velocities are twice as high as those obtained by models. On the other hand, the peculiar nucleus of SwSt1 (PNG 1.5-6.7) exhibits a narrow C III line (see also the $\mathrm{C}$ III profile at high resolution in Grosdidier et al. 2000, Fig. 12) which indicates a velocity of $420 \mathrm{~km} \mathrm{~s}^{-1}$, in good
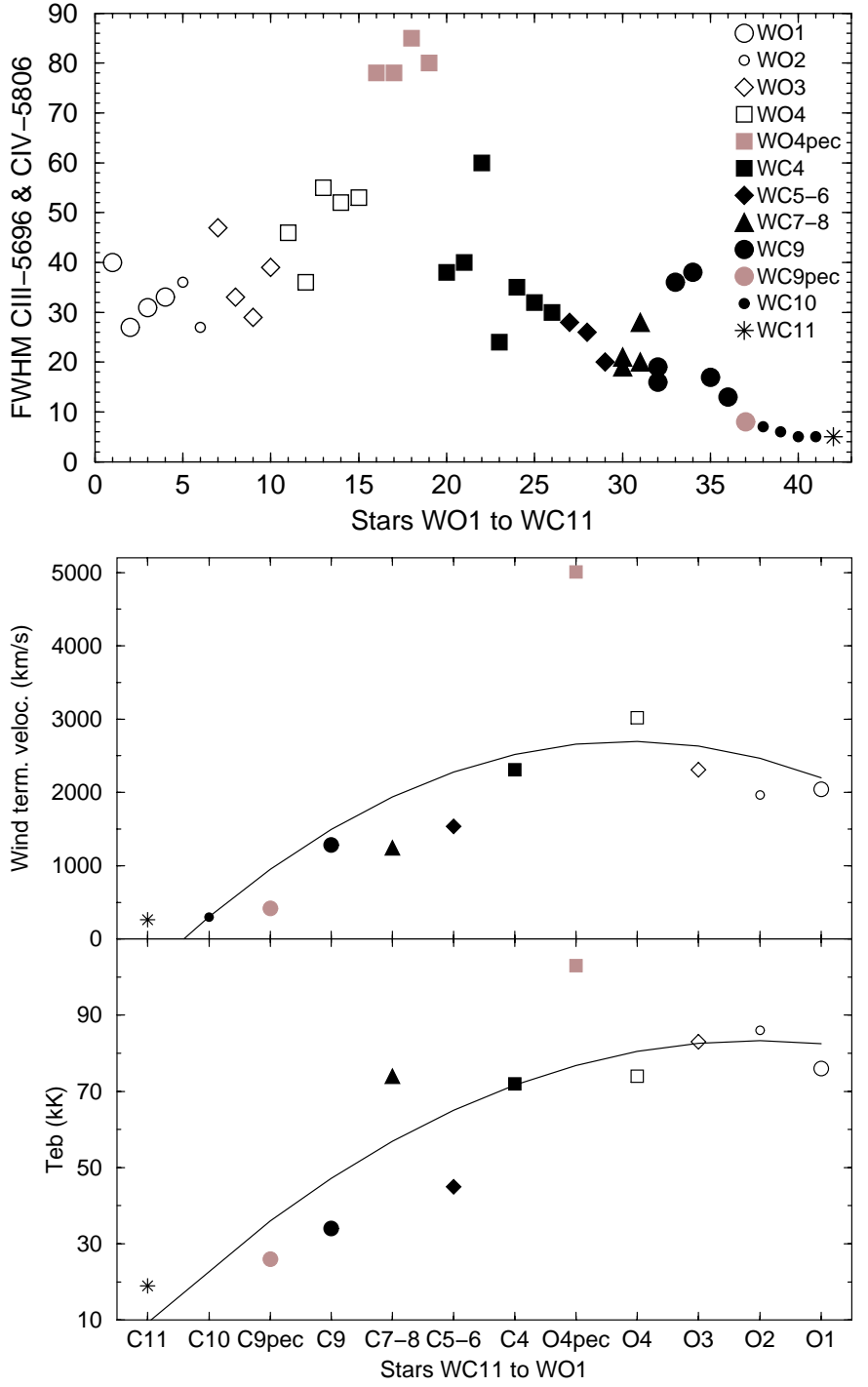

Fig. 8. Upper panel: variation of the $F W H M$ of C IV-5806 and C III5696 along the spectral sequence, averaged from Table 1a. Middle and lower panels: variation of the mean terminal wind velocity and the stellar temperature $\left(T_{\mathrm{eb}}\right)$, respectively, through the spectral sequence from the [WO1-4] to [WC4-11] subtypes. The fits (solid line) include the [WO4]pec point.

agreement with the models by Koesterke \& Hamann (1997b), but which is about half the value obtained by de Marco et al. (2001) by modelling the stellar optical and UV spectra with state-of-the-art non-LTE codes for expanding atmospheres and a composite beta velocity law.

Regular but highly dispersed variations along the [WO]-[WC] sequence are shown by the FWHM of the $\mathrm{C}$ IV and $\mathrm{C}$ III lines with a strong maximum for the [WO4]pecclass (upper panel of Fig. 8). The two measured lines, and particularly the C III-5696 line, are sensitive to wind density and velocity, and therefore show strong variations when the star evolves (Crowther 1999).

Let us recall some properties of the variations of the stellar and nebular parameters. A clear dependance between the terminal velocity and the ionised mass, and also the dynamical age of the nebula is found by Acker et al. (2002). From this 


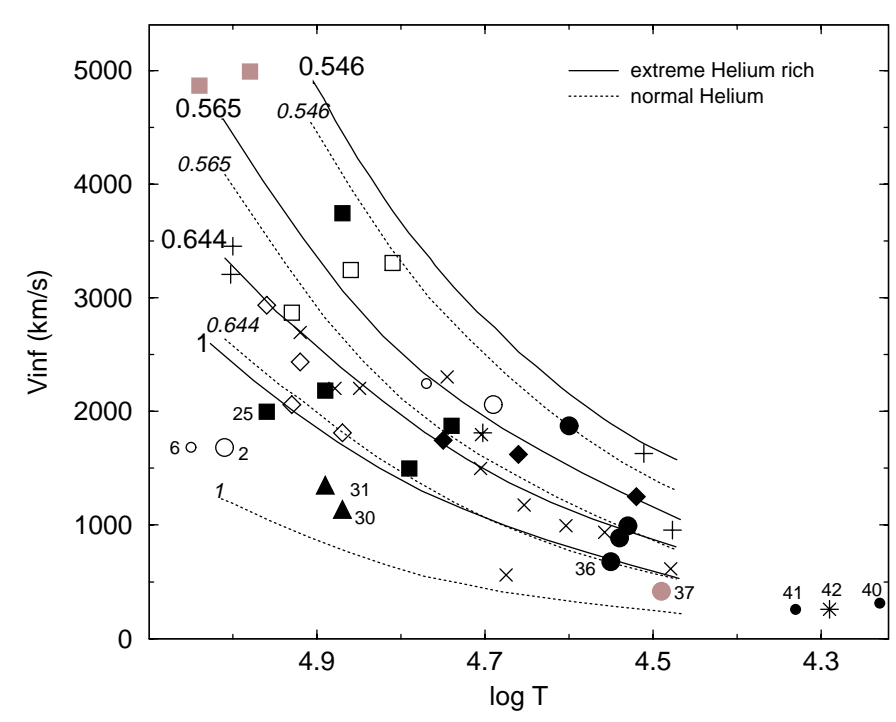

Fig. 9. 42 CSPNe plotted on the tracks from Pauldrach et al. (1988). See the symbol legend in Fig. 8. The data points from Pauldrach et al. (1988) are also shown with the symbols "+" and " $x$ ".

dependence, the mass-loss history for [WC]-type nuclei may be inferred. As the central star evolves from the post-AGB phase to the late [WC] types, its temperature increases, and the density of the fast wind decreases while its terminal velocity increases. This is confirmed by Fig. 8 showing that $V \infty$ increases regularly with the temperature. The very strong dependence of the cooling time on the terminal velocity thus makes the radiative cooling of the shocked fast wind less and less efficient. Therefore, $\mathrm{PNe}$ are expected to initially be momentum-driven and later on become energy-driven, the transition arising for terminal velocities above $\sim 1000 \mathrm{~km} \mathrm{~s}^{-1}$ for $\mathrm{H}$ deficient and metals enriched stellar winds (Mellema 2001). This transition may correspond to the velocity of about $5000 \mathrm{~km} \mathrm{~s}^{-1}$ for the [WO4]pec stars.

\subsection{Estimation of the stellar mass}

The evolutionary tracks of Pauldrach et al. (1988) propose a relation between $V \infty$, temperature and stellar mass. The observed data of our sample indicate dispersed stellar masses around higher values than previous estimations for the CSPNe masses. Improbably high masses are obtained for some WO stars, implying that we probably use underestimated values of $V \infty$ for these very hot CSPNe.

From Table 3 we extract all CSPNe for which the temperature, the $V$ magnitude, the optical extinction coefficient and the distance are known, in order to derive their $\log T$ and $\log L / L_{\odot}$. The HR diagram (Fig. 10) shows that the stars of our sample cluster around the solar mass, like the other PNe. All objects of our sample are concentrated on the constant luminosity track. This could imply that the [WR] phenomenon has generally a short duration. Note that Fig. 10 indicates a high mass for the [WO4pec] star Cn 1-5, the [WC5-6] star M2-20, the [WC8] star NGC 40, the [WC9] star BD +30 3639 and the [WC9pec] star SwSt 1. Figure 9 also indicates a high mass for three of those five stars plus a few others.

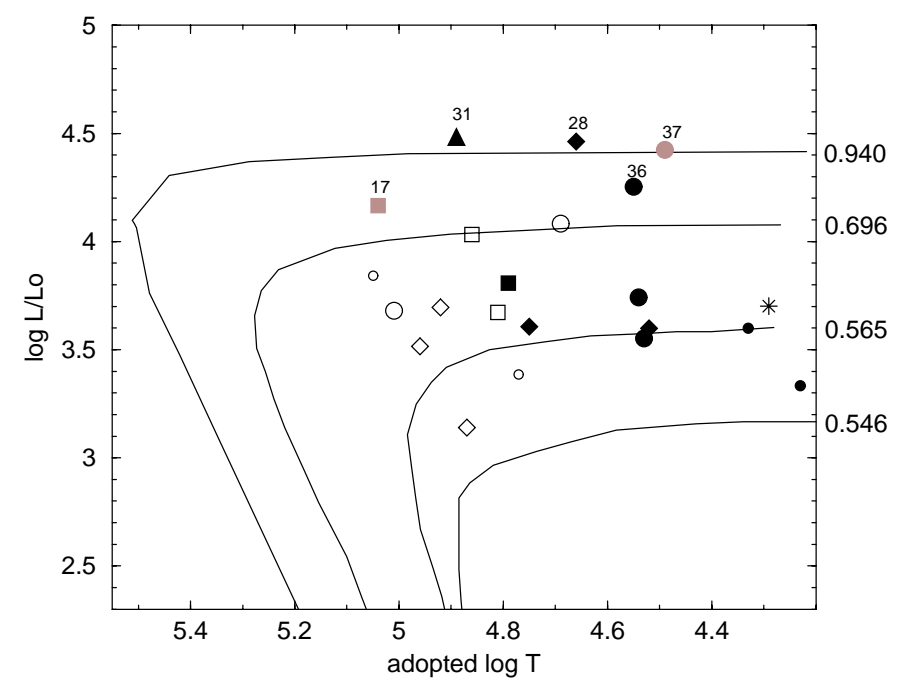

Fig. 10. Positions of the stars on the HR diagram, using symbol legend from Fig. 8. Four evolutionary tracks from Blöcker (1995), for the masses $0.546,0.565,0.696$ and $0.940 M_{\odot}$, are shown in solid lines.

\section{Distribution of the known emission-line galactic CSPNe}

\subsection{The whole set of known emission-line CSPNe}

We surveyed the literature as exhaustively as possible. We also took into account all observations we performed many years ago (see Sect. 2.1).

The list of all known emission-line CSPNe is presented in Table 4, with the following columns:

Column 1: the PN G identification number.

Column 2: the usual name of the PN; He2-436 belongs to the Sagittarius dwarf galaxy (labelled with "S").

Column 3: the spectral class according to the present classification scheme, as given in Table 2. Some spectra observed during our 1994-1996 ESO-runs and of low quality were not shown in Table 1a, but their aspect allows us to propose a spectral class. An asterisk "*” means that the observations were performed in 1993-2000 at OHP. Some results are based on visual inspection on plates obtained in 1974 (marked by “*1”), or from earlier (1961) observations communicated by M. Chopinet (marked by “*2").

Column 4: the spectral class from Tylenda et al. (1993)

Column 5: the spectral types from various papers, in chronological order. References of the papers are given in the caption of the table.

Only a few new objects were discovered very recently. Gorny \& Siodmiak (2002) found 11 new emission-line stars in a study of galactic bulge PNe. In the framework of the follow-up spectroscopy completing Parker's southern $\mathrm{H} \alpha$ survey, 11 new [WC] CSPNe were discovered (9 of them are listed in Table 4), possibly including one WN star (analysis in progress, Parker \& Morgan 2003b).

We exclude V348 Sgr and IRAS 21282+5050 which probably are, respectively, a "peculiar extreme He-star", and a "peculiar late O star" (CMB98). We were not able to classify some low excitation objects: 001.2-3.0, 002.4-03.7, 002.6-03.4 


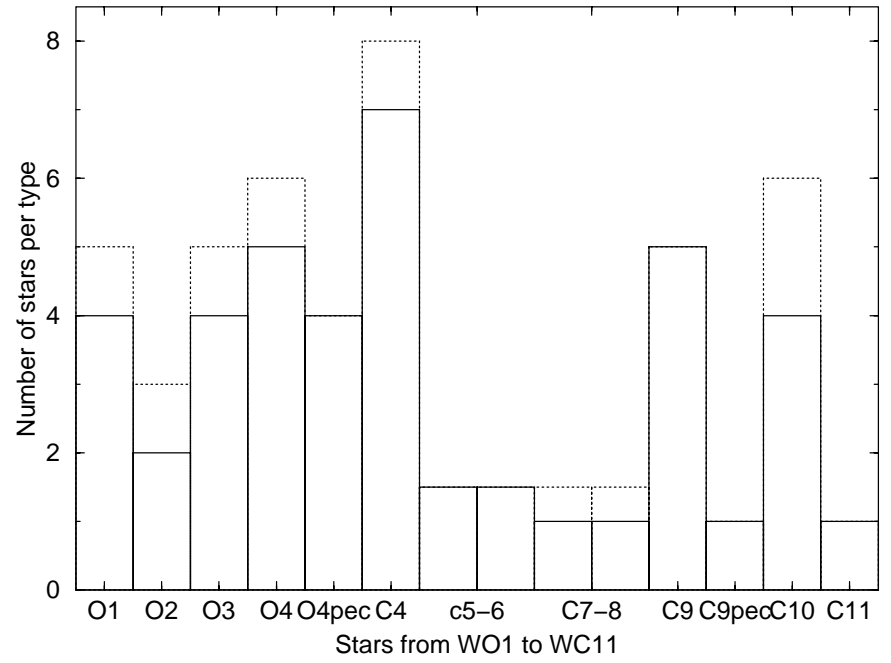

Fig. 11. Histogram of the distribution of our sample (42 stars, solid line), similar to the distribution of the whole well-classified sample (50 stars, dotted line).

and 007.8-04.4. These stars show only narrow and weak C III5696 emission, whereas the C IV-5806, -5471, O V-5595 and the nebular [O III]-5007 lines are absent. Further spectroscopic studies of these stars are needed.

\subsection{The distribution}

We extract from Table 4 all CSPNe having a reliable classification. For the CSPNe we cannot classify we adopt the types from CMB98 or TAS93 (shown in boldface). As seen in Fig. 11, the distribution of our sample is very similar to the distribution of the whole sample, with a peak for [WO4] and [WC4]-type CSPNe.

In our sample we find only 5 CSPNe in the [WC5-8] gap and confirm the relative paucity in this range, possibly related to rapid variations of the wind density (see preceding section). From Table 4 it appears that only 2 CSPNe from this intermediate class belong to the galactic bulge population, and not the majority of these stars as claimed by Gorny \& Siodmiak (2002).

Finally, we note that, as more than $20 \mathrm{CSPNe}$ belong to the galactic bulge, it should be possible to calibrate the distance scale based on the C IV-5806 and C III-5696 lines, in a similar way to that performed by Smith et al. (1990) for massive WC stars. This is a task for a forthcoming paper.

\section{Conclusions}

Based on spectra taken at ESO and OHP, we performed a quantitative classification of $42 \mathrm{WR}$ CSPNe following the ionisation/excitation potential of the lines (Table 1). Our classification is based on a grid of selected line-intensities ordered by decreasing ionisation potential going from 871 to $24 \mathrm{eV}$, appearing like a diagram with two well identified sequences: a series of spectra dominated by the highly ionised oxygen lines (hot [WO1-4] types), and a series dominated by carbon lines (cooler [WC4-11] types). Our scheme, specifically adapted to CSPNe, proposes 20 selected line ratios and the $F W H M$ of C IV and C III lines as diagnostics, which agree well with the 7 line ratios and the FWHM proposed by CMB98.

All subtypes are present in a sequence of decreasing stellar wind temperatures from [WO1] to [WO4] and [WC4] to [WC11], with terminal velocity reaching a maximum value $\left(5000 \mathrm{~km} \mathrm{~s}^{-1}\right)$ for some peculiar [WO4]pec stars. These variations retrace the evolution of the stars along a sequence postAGB - [WC11] to [WC4] - [WO4] to [WO1]. They are related to the evolution of the temperature, but depend essentially on the mass-loss history (Figs. 7 and 8).

The HR diagram and the diagram linking the terminal velocity and the temperature indicate highly dispersed values of the stellar mass for our sample, around a mean mass higher than for normal CSPNe.

The distribution of the 42 stars of our sample shows $24 \%$ [WO1-3], 21\% [WO4] and 17\% [WC4] hot stars, and 26\% cool stars [WC9-11]), the [WC5-8] classes remaining poorly represented (12\%). A similar distribution is shown for the $127 \mathrm{CSPNe}$ with emission-line spectra we compiled from the literature, and which represent $5 \%$ of the whole sample of known PNe.

Acknowledgements. Many thanks to our referee, Albert Zijlstra, for comments and suggestions. A special thank to Björn Stenholm and François Cuisinier, for their faithful help in the ESO observations. We kindly acknowledge Marguerite Chopinet and Quentin Parker for communication of unpublished results, and Mudumba Parthasarathy and Tony Moffat for valuable discussions. This project was partially supported by the Programme National de Physique Stellaire.

\section{References}

Acker, A. 1976, Thesis, Publications de l'observatoire de Strasbourg Acker, A., Ochsenbein, F., Stenholm, B., et al. 1992, 1996, StrasbourgESO catalogue of galactic planetary nebulae, ESO

Acker, A., Gorny, S. K., \& Cuisinier, F. 1996, A\&A, 305, 944

Acker, A., Gesicki, K., Grosdidier, Y., \& Durand, S. 2002, A\&A, 384, 620

Acker, A., \& Grosdidier, Y. 2002, IAU Symp., 209, Canberra, in press Acker, A., \& Durand, S. 2002, IAU Symp., 209, Canberra, in press

Aller, L., \& Liller, W. 1968, in Nebulæ and Interstellar Matter, Vol. VII of Stars and interstellar matter, ed. B. M. Middlehurst, \& L. H. Aller (The University of Chicago Press), 498

Aller, L. 1976, Mem. Soc. R. Sci. Liège 9, 271

Aller, L. H., \& Keyes, C. D. 1985, PASP, 97, 1142

Bensby, T., \& Lunström, I. 2001, A\&AS, 374, 599

Blöcker, T. 1995, A\&A, 299, 755

Cesco, C. U., \& Gibson, J. 1973, A\&AS, 11, 335

Chu, Y. H., Jacoby, G. H., \& Arendt, R. 1987, ApJS, 64, 529

Cohen, M., \& Jones, B. 1987, ApJ, 321, L151

Crowther, P. A. 1999, in WR phenomena in massive stars and starburst galaxies, ed. Van der Hucht, Koenigsberger, \& Eenens, Proceedings IAU Symp., 193, 116

Crowther, P. A., De Marco, O., \& Barlow, M. J. 1998, MNRAS, 296, 367 (CMB98)

Dong, Y. S., \& Hu, J. Y. 1991, Bulletin of Academica Sinica, 11, 172

Dudziak, G., Pequignot, D., Zijlstra, A., \& Walsh, J. R. 2000, A\&A, 363,717

Feibelman, W. A. 1999, ApJ, 514, 296

Gleizes, F., Acker, A., \& Stenholm, B. 1989, A\&A, 222, 237 
Gorny, S. K. 1998, Thesis, Centrum Astronomizne im. Mikolaja Kopernica, Torun

Gorny, S. K. 2001, Ap\&SS, 275, 67

Gorny, S. K., \& Siodmak, N. 2002, IAU Symp., 209, in press

Grosdidier, Y., Acker, A., \& Moffat, A. F. J. 2000, A\&A, 364, 597

Hack, M., \& Struve, O. 1970, Stellar spectroscopy. Vol. 2: Peculiar stars, Trieste Oss. Astr.

Hamann, W. R. 1996a, Ap\&SS, 238, 31

Hamann, W. R. 1996b, ASP Conf. Ser., 96, 127

Heap, S. R. 1982, IAU Symp., 99, 423

Hillier, D. J., \& Miller, D. L. 1999, ApJ, 519, 354

Hu, J. Y., \& Bibo, E. A. 1990, A\&A, 234, 435

van der Hucht, K. A., Conti, P. S., Lundstrom, I., \& Stenholm, B. 1981, SSRv, 28, 227

Kaler, J. B. \& Shaw, R. A. 1984, ApJ, 278, 195

Kaler, J. B., Shaw, R. A., Feibelman, W. A., \& Imhoff, C. L. 1991, PASP, 103, 67

Koesterke, L., \& Hamann, W. R. 1997a, IAU Symp., 180, 114

Koesterke, L., \& Hamann, W. R. 1997b, A\&A, 320, 91

Köppen, J., Durand, S., Acker, A., Parthasarathy, M., \& Stenholm, B. 2002, in preparation

Leuenhagen, U., Heber, U., \& Jeffery, C. S. 1994, A\&AS, 103, 445

Leuenhagen, U., Hamann, W. R., \& Jeffery, S. 1996, A\&A, 312, 167

Leuenhagen, U., \& Hamann, W. R. 1998, A\&A, 330, 265

Liu, X.-W., Storey, P. J., Barlow, M. J., et al. 2000, MNRAS, 312, 585

Lutz, J. H. 1977, ApJ, 211, 469

Lutz, J. H. 1978, IAU Symp., 76, 185

de Marco, O., Storey, P. J., \& Barlow, M. J. 1997, MNRAS, 297, 999

de Marco, O., Crowther, P., Barlow, M. J., et al. 2001, MNRAS, 328, 527

de Marco, O. 2002, IAU Symp., 209, in press

Mellema, G. 2001, Ap\&SS, 275, 147

Méndez, R. H., \& Niemela, V. S. 1979, ApJ, 232, 496
Méndez, R. H., \& Niemela, V. S. 1982, IAU Symp., 99, 457

Méndez, R. H., Kudritzki, R. P., Herrero, A., \& Husfeld, D. N. 1988, A\&A, 190, 113

Méndez, R. H. 1991, IAU Symp., 145, 375

Morgan, D. H., Parker, Q. A., \& Cohen, M. 2003, submitted

Morgan, D. H., Parker, Q. A., \& Russeil, D. 2001, MNRAS, 322, 877

Nugis, T., \& Lamers, H. J. 2000, A\&A, 360, 227

Parker, Q. A. 2003, private communication

Parker, Q. A, \& Morgan, D. H. 2003a, in press

Parker, Q. A., \& Morgan, D. H. 2003b, in preparation

Parker, Q. A. 2002, IAU Symp., 209, Canberra, in press

Pauldrach, A., Puls, J., Kudritzki, R. F., Mendez, R. H., \& Heap, S. R. 1988, A\&A, 207, 123

Parthasarathy, M., Acker, A., \& Stenholm, B. 1998, A\&A, 329, L9

Peña, M., Stasinska, G., \& Medina, S. 2001, A\&A, 367, 983

Preite-Martinez, A., Acker, A., Köppen, J., \& Stenholm, B. 1989, A\&AS, 81, 309

Preite-Martinez, A., Acker, A., Köppen, J., \& Stenholm, B. 1991, A\&AS, 88, 121

Smith, L. F. 1968, MNRAS, 138, 109

Smith, L. F., \& Aller, L. H. 1969, ApJ, 157, 1245

Smith, L. F., Shara, M. M., \& Moffat, A. F. J. 1990, ApJ, 358, 229

Torres, A. V., Conti, P. S., \& Massey, Ph. 1986, ApJ, 300, 379

Van de Steene, G. C., \& Zijlstra, A. A. 1994, A\&AS, 108, 485

Tylenda, R., Acker, A., Stenholm, B., \& Köppen, J. 1992, A\&AS, 95, 337

Tylenda, R., Acker, A., \& Stenholm, B. 1993, A\&AS, 102, 595

Volk, K. M. 1988, private communication

Webster, B. C., \& Glass, I. S. 1974, MNRAS, 166, 491

Webster, B. C. 1975, MNRAS, 173, 437

Zhang, C. Y. 1998, ApJS, 98, 659

Zijlstra, A., \& Walsh, J. 1996, A\&A, 312, L21 